\title{
Le Réseau canadien des forêts modèles et l'Argentine travaillent en équipe au XIIIe Congrès forestier mondial pour fournir un aperçu des recherches sur les indicateurs locaux
}

\author{
by John E. Hall ${ }^{1}$, Sean Dolter ${ }^{2}$ et Monica Gabay ${ }^{3}$
}

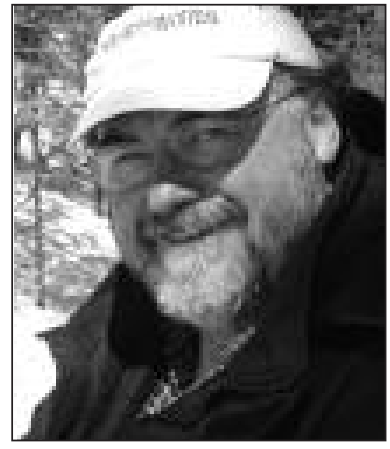

John E. Hall

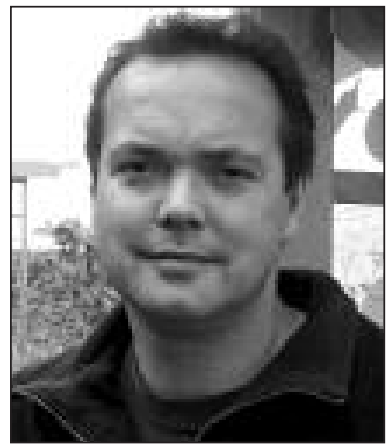

Sean Dolter

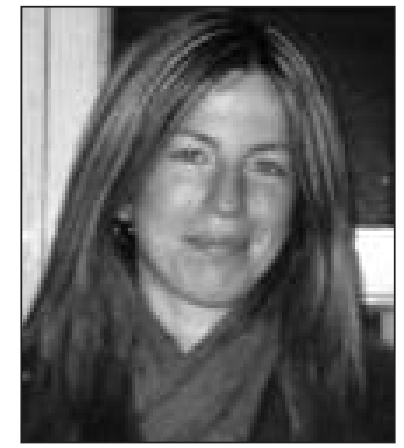

Monica Gabay
Depuis 2 ans, le Réseau canadien des forêts modèles (RCFM), la Forêt modèle de Terre-Neuve et Labrador (FMTNL) et le Service canadien des forêts de Ressources naturelles Canada (SCF-RNCan) collaborent avec le ministère de l'Environnement et du Développement durable de l'Argentine et le Réseau argentin de forêts modèles (RAFM) en vue délaborer des indicateurs locaux de gestion durable des forêts (ADF) pouvant s'appliquer à la situation argentine. Grâce à des ateliers annuels impliquant 6 modèles de forêt argentine, une série d'indicateurs locaux ont été élaborés et sont présentement à l'essai sur le terrain dans les forêts modèles. Des discussions sont en cours avec le Réseau ibéro-américain de forêts modèles afin d'explorer l'expansion du projet à d'autres pays de l'Amérique latine.

Le projet sera présenté dans le cadre d’un événement spécial lors du prochain Congrès forestier mondial qui aura lieu du 18 au 25 octobre à Buenos Aires en Argentine. Les collaborateurs du projet, $\mathrm{D}^{\text {re }}$ Monica Gabay, coordonnatrice du programme du RAFM, M. Sean Dolter, directeur général, FMTL et $\mathrm{D}^{\mathrm{r}}$ John Hall, conseiller scientifique, SCF-RNCan feront la présentation des travaux. Une période de questions suivra.

\section{Bref rappel concernant les critères et indicateurs}

Les critères et indicateurs ( $\mathrm{C}$ et $\mathrm{I}$ ) constituent un cadre qui délimite de façon logique les thèmes regroupés en plusieurs volets parallèles sous le chapitre de l'aménagement durable forestier. Chaque volet divise la chaîne des éléments thématiques en quatre ou cinq grands groupes :

1. Buts

2. Valeurs
3. Indicateurs

4. Objectifs

5. Pratiques (facultatif)

Les indicateurs locaux (IL) sont un sous-ensemble de données mesurables localement à l'intérieur du groupe d'indicateurs du cadre de C et I. Les indicateurs locaux (IL) sont utilisés comme mesures de surveillance et de rapport pour veiller à ce que les objectifs de l'ADF soient atteints de façon efficace dans une région définie.

Le cadre des IL est intégré au système de gestion des forêts pour aider les gestionnaires à mettre en application et à adapter les pratiques en fonction de normes en constante évolution en :

- orientant la façon dont la collecte et la synthèse des données sont effectuées dans les provinces et territoires au fil du temps;

- fournissant un moyen de concilier et de gérer les valeurs et les attentes de nombreux intervenants;

- orientant la façon d'améliorer la pratique par l'application de méthodes de gestion adaptative;

- contribuant à l'uniformisation des processus de collecte des données et de présentation des rapports pour permettre aux administrations de faire un suivi des progrès et d'améliorer les pratiques;

- permettant aux organismes de certification des entreprises forestières de suivre les travaux de l'ADF sur une assise territoriale définie.

\footnotetext{
${ }^{1}$ conseiller scientifique, Ressources naturelles Canada

${ }^{2}$ directeur général, Forêt modèle de Terre-Neuve-et-Labrador

${ }^{3}$ coordonnatrice nationale, Réseau argentin de forêts modèles
} 


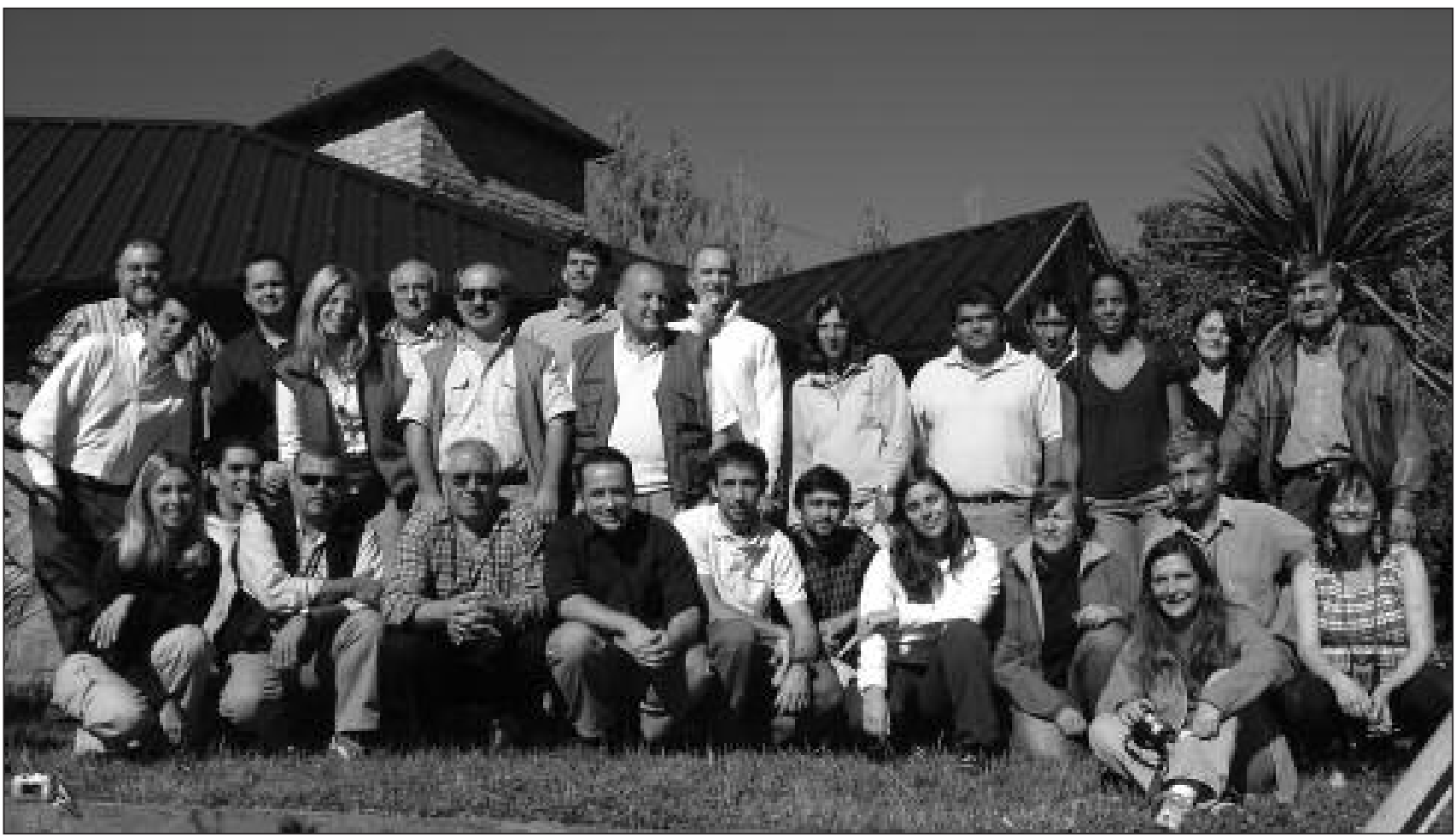

Participants à l'atelier sur les IL des forêts modèles de l'Argentine - Mars 2008

Adoption des C et I - exemples du Canada : au Canada, le cadre de $\mathrm{C}$ et I est largement utilisé et adapté à tous les niveaux pour les nombreux avantages qu'il confère.

\section{En voici quelques exemples :}

Échelle nationale

- Stratégies nationales sur la forêt - production et évaluation

- Rapports sur létat des forêts - collecte et gestion des données

- Liens et contributions avec d'autres initiatives de reddition de comptes fondées sur des indicateurs

- Recherche - cerner les besoins et organiser des programmes

- Appui au commerce international - preuve dengagement envers l'ADF

Échelle provinciale et territoriale

- Stratégies provinciales sur la forêt

- Politiques et réglementation relatives à la forêt

- Responsabilisation sur létat des forêts

Échelle locale

- Permis d'exploitation forestière :

- planification de laménagement forestier et

- certification et vérification des entreprises forestières

- Forêts modèles :

- outil de planification et

- cadre de préparation des rapports sur les progrès en fonction des objectifs de l'ADF

\section{Citations}

"Le Réseau argentin de forêts modèles a maintenant un plan lui permettant dorienter ses forêts modèles vers un jalon unique par le biais duquel tous les sites argentins pourront contribuer au cadre de collecte et de surveillance des données. Ce plan pourra être mis en ouvre à léchelle du site même et influencer la gestion à léchelle nationale. » - Monica Gabay

"Fort de son leadership, expertise et expérience dans le domaine des $C$ et I, le Canada peut contribuer de façon précieuse et soutenue à laménagement durable des forêts de l'Amérique latine et du monde entier. Je suis heureux de prendre part à cette grande initiative. »- John Hall

"En tant que Forêts modèles, nous sommes engagés à partager nos expériences avec nos collègues en Argentine et ailleurs...et vice-versa. Notre expérience au niveau des indicateurs locaux à Terre-Neuve et au Labrador est le résultat d'un travail soutenu impliquant plusieurs de nos partenaires, notons tout particulièrement lengagement de la compagnie Corner Brook Pulp and Paper, des collectivités locales, et des gouvernements du niveau provincial et fédéral. Je suis ravi que notre expérience contribue à développer des stratégies et des pratiques pour l'aménagement durable des forêts à l'extérieur du Canada. »-Sean Dolter 